\title{
GENERALIZED LAPLACE TRANSFORM WITH MATRIX VARIABLES
}

\author{
R.M. JOSHI and J.M.C. JOSHI \\ Department of Mathenatics \\ Govt. P. G. College \\ Pithoragarh (U.P.) \\ INDIA \\ (Received September 9, 1985)
}

ABSTRACT. In the present paper we have extended generalized Laplace transforms of Joshi to the space of $\mathrm{m} \times \mathrm{m}$ symmetric matrices using the confluent hypergeometric function of matrix argument defined by Herz as kernel. Our extension is given by

$$
g(Z)=\frac{\Gamma_{m}(\alpha)}{\Gamma_{m}(\beta)} \int_{\wedge>0} F_{1}(\alpha: \beta:-\wedge Z) f(\wedge) d \wedge
$$

The convergence of this integral under various conditions has also been discussed. The real and complex inversion theorems for the transform have been proved and it has also been established that Hankel transform of functions of matrix argument are limiting cases of the generalized Laplace transforms.

KEY WORDS AND PHRASES. Integral transforms, Laplace transform, Hankel transform of Herz, functions of matrix argument.

1980 AMS SUBJECT CLASSIFICATION CODES. $44 F 12$.

\section{INTRODUCTION.}

A function of matrix argument is a real or complex valued function of the elements of a matrix. Let $\Lambda$ be a symmetric matrix of dimension $m \times m$. The function $f(A)$ is called symmetric function if $f(\Lambda)=f\left(O \wedge O^{\prime}\right)$, where $0 \in 0_{m}$ : the group of $m \times m$ orthogonal matrices. If $f(\Lambda)$ is a symmetric function, then it is a function of the elementary functions like trace, determinant, etc., of $\Lambda$. Herz [1] has defined the Laplace transform with matrix variables by

$$
g(Z)=\underset{\wedge>0}{\int} \operatorname{etr}(-\wedge Z) \mathrm{f}(\wedge) \mathrm{d} \wedge,
$$

where $\wedge \in \mathrm{S}_{\mathrm{m}}$; the space of $\mathrm{m} \times \mathrm{m}$ real symmetric matrices parameterized by $\left(\lambda_{t j}\right)$, $\mathrm{Z}=\mathrm{X}+\mathrm{IY}, \mathrm{X}, \mathrm{Y} \in \mathrm{S}_{\mathrm{m}}^{*}$; the space of $\mathrm{m} \times \mathrm{m}$ real symmetric matrices parameterized by $x=\left(n_{i j} x_{i j}\right) ; \quad n_{i j}$ being 1 if $1=j, 1 / 2$ otherwise. Also $d \wedge=\prod_{i \leq j} d \lambda_{i j}$ is the Lebesgue measure in $s_{m}$, etr $(\Lambda)$ stands for $e^{\operatorname{trace} \wedge}$. The integration in (1.1) is over the set of all positive definite $\wedge$. The inverse transform (1.1) is given by

$$
\frac{1}{(2 \pi i)^{n}} \int_{\operatorname{Re}(Z)=X_{0}>0} \operatorname{etr}(\wedge Z)_{g}(Z) d Z=\left\{\begin{array}{l}
f(\Lambda j, \wedge>0 ; \\
0, \text { otherwise }
\end{array}\right.
$$


Herz [1] has used (1.1) and (1.2) in defining hypergeometric functions of matrix argument. The confluent hypergeometric function, ${ }_{1} F_{1}$, is defined by

$$
{ }_{1} F_{1}(a ; b ; M)=\frac{\Gamma_{m}(b)}{(2 \pi i)^{n}} \int_{\operatorname{Re}(Z)=X_{0}>0} \operatorname{etr}(Z) \operatorname{det}(E-M Z)^{-a}(\operatorname{det} Z)^{-b} d Z,
$$

which holds for arbitrary complex $M$ and $a, \operatorname{Re}(b)>m$, provided we take $X_{0}>\operatorname{Re}(M)$. Here $\Gamma_{m}(b)$ is the generalized gamma function of Siegel, and $p=(m+1) / 2$.

Herz [1] has shown that

$$
{ }_{1} F_{1}(a ; b ; Z)=\frac{\Gamma_{m}(b)}{\Gamma_{m}(a) \Gamma_{m}(b-a)} \int_{0}^{E} \operatorname{etr}(Z R)(\operatorname{det} R)^{a-p} \operatorname{det}(E-R)^{b-a-p} d R,
$$

which holds for $\operatorname{Re}(a), \operatorname{Re}(\mathrm{b}) \operatorname{Re}(\mathrm{b}-\mathrm{a})>\mathrm{p}-1$.

In the present paper, we shall extend the generalized Laplace transform of Joshi [2], to the space of $\mathrm{m} \times \mathrm{m}$ real symmetric matrices. The kernel will be ${ }_{1} \mathrm{~F}_{1}$ function of matrix argument. We shall also prove some theorems on the properties of the transform thus defined. In section 3, we shall establish a relation between Laplace transform and generalized Laplace transform through the operator of fractional integration defined by Garding [3];

$$
I^{a} f(\wedge)=\frac{1}{\Gamma_{m}(a)} \int_{0}^{\wedge} f(R) \operatorname{det}(\wedge-R)^{a-p} d R,
$$

holding under suitable conditions on the function $f(R)$. Integral (1.5) is a generalization of Riemann Liouville integral to matrix variables.

2. DEFINITION.

Joshi [2] has defined the generalized Laplace transform in the scalar case by

$$
g(x)=\frac{\Gamma(b+c+1)}{\Gamma(a+b+c+1)} \int_{0}^{\infty}(x y)^{b}{ }_{1} F_{1}(b+c+1 ; a+b+c+1 ;-x y) f(y) d y
$$

In analogy with (1.1), we consider the relation

$$
g(z)=\frac{\Gamma_{m}(a)}{\Gamma_{m}(b)} \int_{\wedge>0} F_{1}(a ; b ;-\wedge z) f(\wedge) d \wedge ; z=x+1 y,
$$

where $\wedge \in \mathrm{S}_{\mathrm{m}}, \mathrm{X}, \mathrm{y} \in \mathrm{S}_{\mathrm{m}}^{*}$, and ${ }_{1} \mathrm{~F}_{1}$ is the function defined by (1.3). If Integral (2.2) converges in some $Z$ region, then it represents a function, $g(Z)$, of the elements of the complex symmetric matrix $Z$ there. In this case, $g(Z)$ will be called generalized Laplace transform of $f(\wedge)$.

Now we have to establish the convergence of (2.2) to find the region where it defines a function. But, before proving the convergence theorems, we have to say something on the limit concept in matrix space.

We know that the space $S_{m}$ is a partially ordered set for the order relatioi, ">" defined in it by $A>B \Leftrightarrow A-B$ is positive definite, where $A, B \in S_{m} \cdot S_{m}$ is also a directed set since for any pair of matrices $A, B \in S_{m}$ there exists a number $k$ such that $A<k E$ and $B<k E$, $E$ being $m \times m$ unit matrix.

For any two functions $f(\Lambda)$ and $g(\Lambda)$ defined in $s_{m}$, we will say that $f(\Lambda)$ is of the order of $g(\wedge)$ for infinitely large $\wedge$ (in the sense of the order relation; >), and will write

$$
f(\Lambda) \sim g(\Lambda) ; \quad \wedge \rightarrow(\infty),
$$


If Moore-Smith limit (Yoshida [5], p. 103) of $f(\wedge) / g(\wedge)$ through the directed set $s_{m}$ is equal to 1 .

3. CONVERGENCE THEOREMS.

Before proving the theorems on convergence of the integral (2.2), we will prove a few lemmas which will be useful in proving convergence theorems.

LEMMA 1 (A) If $\operatorname{Re}(\mathrm{b})>\mathrm{m}$, and $\operatorname{Re}(\mathrm{b}-\mathrm{a})>\mathrm{p}-1$,

$$
{ }_{1} \mathrm{~F}_{1}(\mathrm{a} ; \mathrm{b} ;-\mathrm{M}) \sim \frac{\Gamma_{\mathrm{m}}(\mathrm{b})}{\Gamma_{\mathrm{m}}(\mathrm{b}-\mathrm{a})}(\operatorname{det} \mathrm{M})^{-\mathrm{a}} ; \mathrm{M} \rightarrow(\infty) \text {. }
$$

(B) If $\operatorname{Re}(\mathrm{b})>\mathrm{m}$, and $\operatorname{Re}(\mathrm{a})>\mathrm{p}-1$,

$$
{ }_{1} F_{1}(a ; b ; M) \sim \frac{\Gamma_{m}(b)}{\Gamma_{m}(a)} \operatorname{etr}(M)(\operatorname{det} M)^{-(b-a)} ; M \rightarrow(\infty) \text {. }
$$

We have, from (1.3),

$$
\begin{aligned}
& { }_{1} F_{1}(a ; b ;-M)=\frac{\Gamma_{m}(b)}{(2 \pi i)^{n}} \int_{\operatorname{Re}(Z)}^{\operatorname{etr}}(Z)=X_{0} \operatorname{det}\left(E-M z^{-1}\right)^{-a}(\operatorname{det} Z)^{-b} d Z ; \operatorname{Re}(b)>m \\
& =\frac{\Gamma_{m}(b)}{(2 \pi i)^{n}}(\operatorname{det} M)^{-a} \int_{\operatorname{Re}(Z)=X_{0}}^{\operatorname{etr}(Z)}(\operatorname{det} Z)^{-(b-a)} \operatorname{det}\left(E-Z M^{-1}\right)^{-a} d Z
\end{aligned}
$$

Now, if $M \rightarrow(\infty)$, applying formula (1.1) of Herz [1], we obtain

$$
{ }_{1} F_{1}(a ; b ;-M)(\operatorname{det} M)^{a}=\frac{\Gamma_{m}(b)}{\Gamma_{m}(b-a)} ; \operatorname{Re}(b-a)>p-1 .
$$

This proves part (A) of the lemma.

To prove the part (B), we use Kummer's formula (Herz [1]):

$$
{ }_{1} \mathrm{~F}_{1}(\mathrm{a} ; \mathrm{b} ; \mathrm{M})=\operatorname{etr}(\mathrm{M}){ }_{1} \mathrm{~F}_{1}(\mathrm{~b}-\mathrm{a} ; \mathrm{b} ;-\mathrm{M}) \text {. }
$$

Using part (A) of the lemma, and applying Kummer's formula, part (B) of the lemma is proved.

LEMMA 2.

$$
\int_{\wedge>0} F_{1}(a ;, b ;-\wedge \mathrm{Z})(\operatorname{det} \wedge)^{\delta-p} d \Lambda=\frac{\Gamma_{m}(b) \Gamma_{m}(\delta) \Gamma_{m}(a-\delta)}{\Gamma_{m}(a) \Gamma_{m}(b-\delta)} \cdot(\operatorname{det} z)^{-\delta}
$$

where $\operatorname{Re}(a), \operatorname{Re}(b), \operatorname{Re}(\delta), \operatorname{Re}(b-a), \operatorname{Re}(b-\delta), \operatorname{Re}(a-\delta)>p-1$, and $\operatorname{Re}(Z)>0$.

From (1.4), we have

$$
\frac{\Gamma_{m}(a) \Gamma_{m}(b-a)}{\Gamma_{m}(b)}{ }_{1} F_{1}(a ; b ;-Z)=\int_{0}^{E} \operatorname{etr}(-R \wedge Z)(\operatorname{det} R)^{a-p} \operatorname{det}(E-R)^{b-a-p} d R ;
$$

where for $\operatorname{Re}(a), \operatorname{Re}(b), \operatorname{Re}(b-a)>p-1$.

Substituting the value of ${ }_{1} F_{1}(a, b, \wedge z)$ in the left hand side of $(3.3)$, and changing the order of integration, we have

$$
\int_{\wedge>0}{ }_{1} F_{1}(a ; b ;-\wedge z)(\operatorname{det} \wedge)^{\delta-p} d \wedge=\frac{\Gamma_{m}(b) \Gamma_{m}(\delta)}{\Gamma_{m}(a) \Gamma_{m}(b-a)} \cdot \beta_{m}(a-\delta, b-a)(\operatorname{det} z)^{-\delta},
$$

where $\beta_{m}(a-\delta, b-a)$ is the generalized beta function of Siegel and

$$
\beta_{m}(a-\delta, b-a)=\frac{\Gamma_{m}(a-\delta) \Gamma_{m}(b-a)}{\Gamma_{m}(b-\delta)} \text {. }
$$

This proves the lemma. Now we prove:

THEOREM 1. (a) If the integral (2.2) converges absolutely for $Z=Z_{0}$, for which $\operatorname{Re}\left(z_{0}\right)<0$, then 1 t converges absolutely for every $Z$ for which $\operatorname{Re}(Z)>0$ provided 
$\operatorname{Re}(\mathrm{b})>\mathrm{m}, \operatorname{Re}(\mathrm{b}-\mathrm{a})>\mathrm{p}-1$.

(b) If the integral (2.2) converges absolutely for $z=z_{0}$ where $\operatorname{Re}\left(z_{0}\right)<0$, then it converges absolutely for every $Z$ for $\operatorname{Re}\left(Z_{0}\right)<\operatorname{Re}(Z)<0$, provided $\operatorname{Re}(a)>p-1$, $\operatorname{Re}(\mathrm{b})>\mathrm{m}$.

(c) If $a=b$, and (2.2) converges absolutley for $z=z_{0}$, then it converges for every $Z$ for which $\operatorname{Re}(Z)>\operatorname{Re}\left(Z_{0}\right)$.

PROOF. We have

$$
\int_{\wedge>0}\left|F_{1} F_{1}(a ; b ;-\wedge) f(\wedge)\right| d \wedge=\int_{\Lambda>0} \frac{{ }_{1} F_{1}(a ; b ;-Z)}{{ }_{1} F_{1}(a ; b ;-Z)} \mid{ }_{1} F_{1}\left(a ; b ;-\wedge Z_{0}\right) f(\wedge) d \wedge
$$

Now, as $\wedge \rightarrow(\infty)$ from lemma 1 , we have

$$
\left|\frac{\mathrm{F}_{1}(\mathrm{a} ; \mathrm{b} ;-N \mathrm{Z})}{\mathrm{F}_{1}\left(\mathrm{a} ; \mathrm{b} ;-\mathrm{N}_{0}\right)}\right| \sim\left|\frac{(\operatorname{det} N)^{-a}}{\left(\operatorname{det} \mathrm{Z}_{0}\right)^{-\mathrm{a}}}\right|=\left|\left(\operatorname{det} \mathrm{Z}_{0} \mathrm{z}^{-1}\right)^{\mathrm{a}}\right| \text {, }
$$

provided we have $\operatorname{Re}(\mathrm{b})>\mathrm{m}, \operatorname{Re}(\mathrm{b}-\mathrm{a})>\mathrm{p}-1, \operatorname{Re}(\mathrm{Z}), \operatorname{Re}\left(\mathrm{Z}_{0}\right)>0$. But ${ }_{1} \mathrm{~F}_{1}(\mathrm{a} ; \mathrm{b} ;-\wedge \mathrm{Z})$ is bounded in the set of positive definite $\wedge$, and does not vanish anywhere there, provided $\operatorname{Re}(\mathrm{b})>\mathrm{m}, \operatorname{Re}(\mathrm{b}-\mathrm{a})>\mathrm{p}-1$, and $\operatorname{Re}(\mathrm{z})>0$. Thus we see that

$$
\frac{{ }_{1} F_{1}(a ; b ;-\wedge z)}{F_{1}\left(a ; b ;-\wedge Z_{0}\right)}
$$

is bounded in the set of p.d. $\wedge$, provided $\operatorname{Re}(b)>m, \operatorname{Re}(b-a)>p-1$, and $\operatorname{Re}(z)>0$. Now (3.4) reduces to

$$
\int_{\wedge>0}\left|F_{1} F_{1}(a ; b ;-\wedge Z) f(\wedge)\right| d \wedge \leq\left. k \cdot \int\right|_{1} F_{1}\left(a ; b ;-\wedge Z_{0}\right) f(\wedge) \mid d \wedge
$$

where $k$ is the least upper bound (1.u.b.) of

$$
\frac{{ }_{1} F_{1}(a ; b ;-N)}{{ }_{1} F_{1}\left(a ; b ;-N Z_{0}\right)} \text {. }
$$

This proves part (a) of the theorem.

Now, if $\operatorname{Re}(Z)<0, \operatorname{Re}\left(Z_{0}\right)<0, \operatorname{Re}(b)>m, \operatorname{Re}(a)>p-1$, we have from 1 emma $1(b)$,

$$
\left|\frac{{ }_{1} F_{1}(a ; b ;-\wedge Z)}{F_{1}\left(a ; b ;-\wedge Z_{0}\right)}\right| \sim\left|\operatorname{etr}\left(-\wedge\left(Z-Z_{0}\right)\right)\left(\operatorname{det} Z_{0} Z^{-1}\right)^{b-a}\right| \text {; }
$$

So, as in the proof of part (a) of the theorem, we once again note that

$$
\frac{1 F_{1}(a ; b ;-\wedge Z)}{{ }_{1} F_{1}\left(a ; b ;-\wedge Z_{0}\right)}
$$

is a bounded function in the partially ordered set, $\wedge$, provided $\operatorname{Re}(b)>m, \operatorname{Re}(a)$, $\operatorname{Re}(b-a)>p-1$. So, it follows from inequality (1.4), that the integral (2.2) is absolutely convergent. This proves part (b) of the theorem.

We have yet to discuss the case $a=b$. In this case, $F_{1}(a ; b ;-\wedge Z)$ is equal to $\operatorname{etr}(-\wedge Z)$, so that the transform (2.2) is reduced to the Laplace transform (1.1) which is absolutley convergent for $a 11 \mathrm{Z}$ for which $\operatorname{Re}(\mathrm{Z})>\operatorname{Re}\left(\mathrm{z}_{0}\right)$, provided the integral (2.2) is absolutely convergent for $Z=z_{0}$. This proves part (c) of the theorem.

THEOREM 2. If $f(\wedge)$ is absolutely integrable in the partially ordered set $\wedge$ i.e., 


$$
\underset{\Lambda>0}{\int}|\mathrm{f}(\Lambda)| \boldsymbol{d}<\infty
$$

then the integral (2.2) is absolutely convergent for all $\mathrm{Z}$, for which $\operatorname{Re}(\mathrm{Z})>0, \operatorname{Re}(\mathrm{a})$, $\operatorname{Re}(b), \operatorname{Re}(b-a)>p-1$.

PROOF. Since ${ }_{1} F_{1}(a ; b ;-\wedge Z)$ is bounded in the partially ordered set $\wedge$ provided $\operatorname{Re}(\mathrm{z})>0, \operatorname{Re}(\mathrm{a}), \operatorname{Re}(\mathrm{b}), \operatorname{Re}(\mathrm{b}-\mathrm{a})>\mathrm{p}-1$, therefore we have

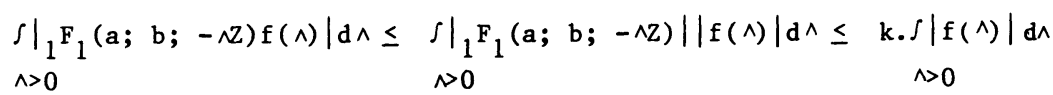

where $k$ is $1 . u . b$. of ${ }_{1} F_{1}(a ; b ;-\wedge Z)$ in the partially ordered set $\wedge$ for $\operatorname{Re}(Z)>0$. This proves the theorem.

THEOREM 3. If, for some number $d$ and a positve number $k$,

$$
|f(\wedge)|<k\left|(\operatorname{det})^{\mathrm{d}-\mathrm{p}}\right| ; \wedge>0,
$$

then the integral (2.2) is absolutely convergent for $\operatorname{Re}(Z)>0$, provided $\operatorname{Re}(a), \operatorname{Re}(b)$, $\operatorname{Re}(b-a), \operatorname{Re}(a-d), \operatorname{Re}(b-d)>p-1$.

PROOF. We have, from lemma 2 ,

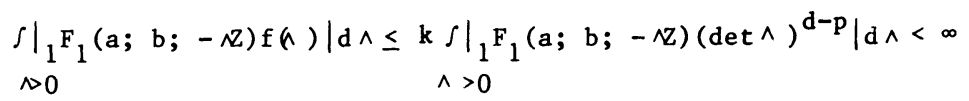

where $\operatorname{Re}(a), \operatorname{Re}(b), \operatorname{Re}(b-a), \operatorname{Re}(a-d), \operatorname{Re}(b-d)>p-1$, and $\operatorname{Re}(z)>0$. This proves the theorem.

THEOREM 4. If, for any $m \times m$ real symmetric matrix $R$,

$$
|f(\Lambda)|<k \cdot \operatorname{etr}(-R \wedge)\left|(\operatorname{det} \wedge)^{b-p}\right|
$$

then the integral (2.2) is absolutely convergent for positive definite $R$ provided $\operatorname{Re}(\mathrm{Z}+\mathrm{R})>0, \operatorname{Re}(\mathrm{b})>\mathrm{p}-1$.

PROOF.

$$
\begin{aligned}
&\left.\int\right|_{1} F_{1}(a ; b ; \wedge Z) f(\wedge) \mid d^{\wedge} \leq\left.\int\right|_{\substack{\wedge>0 \\
1>0}} F_{1}(a ; b ;-\wedge Z)|| f(\wedge) \mid d \wedge< \\
&<\left.k \int\right|_{\substack{1 \\
\wedge>0}} F_{1}(a ; b ;-\wedge Z) \text { etr }(-R \wedge)(\operatorname{det} \wedge)^{b-P} \mid d \wedge<\infty,
\end{aligned}
$$

from Herz ([1], formula (2.7)) provided we have $\operatorname{Re}(\mathrm{Z}+\mathrm{R})>0, \operatorname{Re}(\mathrm{R})>0, \operatorname{Re}(\mathrm{b})>\mathrm{p}-1$. This proves the theorem.

4. GARDING'S FRACTIONAL INTEGRAL AND GENERALIZED LAPLACE TRANSFORM.

In this section, we have derived certain connections between Laplace transform, Hankel transform, and generalized Laplace tranform with the help of Garding's fractional integral.

From formula (1.4), we can show, by certain change of variables, that

$$
\frac{\Gamma_{m}(a)}{\Gamma_{m}(b)} \cdot{ }_{1} F_{1}(a ; b ;-\wedge Z)(\operatorname{det} Z)^{b-p}=\frac{1}{\Gamma_{m}(b-a)} \int_{0}^{Z} \operatorname{etr}\left(-R \wedge(\operatorname{det} R)^{a-p} \operatorname{det}(Z-R)^{b-a-p_{d R}}\right.
$$

where $\operatorname{Re}(a), \operatorname{Re}(b), \operatorname{Re}(b-a)>p-1$.

THEOREM 5. If

and

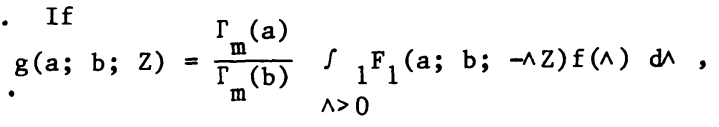

$$
\phi(Z)=\int_{\wedge>0} \operatorname{etr}(-\wedge Z)(\operatorname{det} z)^{a-p_{f}(\wedge) d \Lambda}
$$


then

$$
\mathrm{I}^{\mathrm{b}-\mathrm{a}} \phi(\mathrm{Z})=(\operatorname{det} \mathrm{Z})^{\mathrm{b}-\mathrm{p}} \mathrm{g}(\mathrm{a} ; \mathrm{b} ; \mathrm{z})
$$

Provided integrals $(4.1)$ and (4.2) converge in the generalized right half plane $\operatorname{Re}(z)>0$, and $\operatorname{Re}(a), \operatorname{Re}(b-a)>p-1$.

PROOF. Applying the operator of fractional integration to both the sides of (4.3), we have by changing the order of integration which is justified by Fubini's theorem

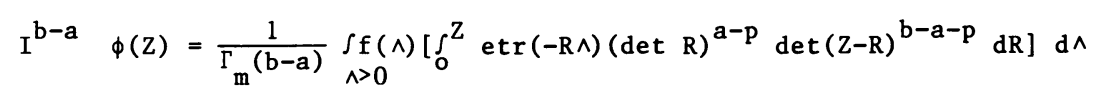

Now, from (4.1) we have in view of (4.2),

$$
I^{b-a} \phi(Z)=(\operatorname{det} z)^{b-p} g(a ; b ; z) .
$$

This proves the theorem.

From (4.2) and (4.3) we note that $\phi(Z)=g(a ; a ; z)(\operatorname{det} Z)^{a-p}$, since ${ }_{1} F_{1}(a ; a ;-\wedge Z)=\operatorname{etr}(-\wedge Z)$.

From Garding's [3] theory, if derivatives of a function, $f(\wedge)$ exist for sufficiently high orders,

$$
D^{a} I^{a} f(\Lambda)=f(\Lambda) \text {. }
$$

Since $\phi(Z)$ in equation (4.3) is a Laplace transform, the derivatives of it will exist for all orders. So from $(4.4)$, we have

$$
\phi(z)=g(a ; a ; z)(\operatorname{det} z)^{a-p}=D^{b-a}\left[(\operatorname{det} z)^{b-p} g(a ; b ; z)\right] .
$$

where $D^{b-a}$ is the differential operator of fractional order (Garding [3]). Moreover, for $\wedge \in S_{m}$,

$$
D=\operatorname{det}\left(n_{i j} \frac{\partial}{\partial \lambda_{i j}}\right),
$$

$n_{i j}$ being 1 if $i=j$, and $1 / 2$ otherwise. And for $z \in S_{m}^{*}$,

$$
D_{z}=\operatorname{det}\left(\frac{\partial}{\partial z_{i j}}\right) \text {. }
$$

Formula (4.5) has been established for real $z$, it follows for all complex $z$ for which $\operatorname{Re}(Z)>0$ by analytic continuation. But we must note that in any case (b-a) is a non negative integer greater than $\mathrm{p}-1$, and $\operatorname{Re}(\mathrm{a})>\mathrm{p}-1$. But the condition;

$(b-a)>p-1$, can be relaxed to the condition $(b-a)>0$. To do so, we apply D-operator to both the sides of (4.2) after multiplying it by (det $z)^{b-p}$ to get (4.5), but then we must have; $\operatorname{Re}(a)>m$, and $b-a$ should be a non negative integer.

Formula (4.5) allows us to find a complex inversion theorem for the generalized Laplace transform.

THEOREM 6. If, for $\operatorname{Re}(a), \operatorname{Re}(b)>m$,

$$
\frac{\Gamma_{m}(a)}{\Gamma_{m}(b)} \cdot \int_{\Lambda} F_{1}(a ; b ; \wedge Z) f(h) d \Lambda=g(a ; b ; Z)
$$

is absolutely convergent in the generalized right half plane given be $\operatorname{Re}(Z)>x_{0}$, and

$$
\int_{Y \in S_{m}^{*}, X>X_{0}}\left|\operatorname{det}(X+i Y)^{b-p} g(a ; b ; X+i Y)\right| d Y<\infty,
$$




$$
\lim _{X \rightarrow(\infty)} \iint_{Y \in S_{m}^{*}} \operatorname{det}(X+1 Y)^{b-p} g(a ; b ; X+1 Y) \mid d Y=0
$$

then, for $\Lambda>0$,

$$
\left.\frac{(-1)^{m(b-a)} \Gamma_{m}(b-a+p)}{(2 \pi i)^{n} \Gamma_{m}(b)} \cdot \int_{\Lambda>0} F_{1}(b-a+p) b ; \wedge z\right)(\operatorname{det} \wedge z)^{b-p} g(a ; b ; z) d z=f(\Lambda) .
$$

provided $(b-a)$ is a non negative integer.

PROOF. Under the conditions of the theorem, (det $z)^{b-p} g(a ; b ; Z)$ can be represented as a Laplace transform of a function, say $\psi(\wedge)$, so that we have

$$
(\operatorname{det} Z)^{\mathrm{b}-\mathrm{p}} \mathrm{g}(\mathrm{a} ; \mathrm{b} ; \mathrm{Z})=\int_{\Lambda>0} \operatorname{etr}(-\wedge \mathrm{Z}) \psi(\Lambda) \mathrm{d} \Lambda
$$

Now, applying D-operator to both the sides of (4.6), we have

$$
D^{b-a}\left[(\operatorname{det} Z)^{b-p} g(a ; b ; Z)\right]=\int_{\Lambda>0} \operatorname{etr}(-\wedge Z)(\operatorname{det}-\wedge)^{b-a} \psi(\wedge) d \wedge
$$

Now, from Herz([1]), formula $(1.1))$, (det $z)^{-a-p}$ is the Laplace transform

of $\frac{1}{\Gamma_{m}(a-p)} \cdot(\text { det })^{a-2 p}$, provided $\operatorname{Re}(a)>2 p-1=m$. So, from the convolution property of Laplace transform, (det $z)^{-a-p} D^{b-a}\left[(\operatorname{det} z)^{b-p} g(a ; b ; z)\right]$ is the Laplace transform of

$$
f(\Lambda)=I^{a-p}\left[\operatorname{det}(-\Lambda)^{b-a} \psi(\Lambda)\right]
$$

Now, inverting (4.6) by complex inversion formula of Laplace transform, (Herz [1]), which is permissible under the conditions of the theorem, and then putting the values of $\psi(\Lambda)$ in $(4.7)$, we obtain

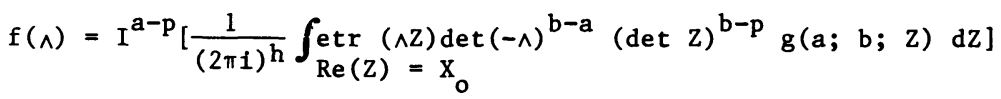

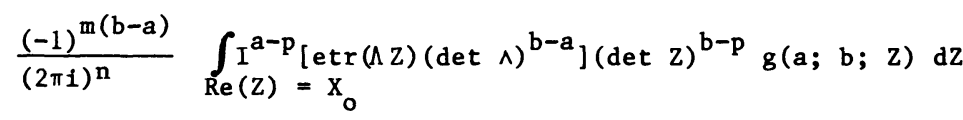

Now, from (4.1), we have

This proves the theorem.

$$
f(\wedge)=\frac{(-1)^{m(b-a)}}{(2 \pi i)^{n}} \frac{\Gamma_{m}(b-a+p)}{\Gamma_{m}(b)} \int_{\operatorname{Re}(Z)=X_{0}} F_{1}(b-a+p ; b ; \wedge Z)(\operatorname{det} \wedge Z)^{b-p} g(a ; b ; Z) d Z
$$

COROLLARY. If the conditions of Theorem 6 hold, $b-a=0$ and

$$
g(Z)=\int_{\wedge>0}^{\operatorname{etr}(-\wedge Z) f(\wedge) d \wedge,}
$$

then

$$
f(\wedge)=\frac{\Gamma_{m}(p)}{\Gamma_{m}(b)(2 \pi 1)^{n}} \quad \int_{\operatorname{Re}(Z)=x_{0}} F_{1}(p ; b ; \wedge Z)(\operatorname{det} \wedge Z)^{b-p} g(Z) d Z
$$

This corollary can be deduced by noting the simple fact that

$$
{ }_{1} F_{1}(b ; b ; Z)=\operatorname{etr}(Z) \text {. }
$$

This corollary gives a new inverison fromula for the Laplace transform with matrix 
variables. In the scalar case; $m=1$, formula (4.8) reduces to Roony's [4] formula.

5. HANKEL TRANSFORM AND GENERALIZED LAPLACE TRANSFORM.

Herz [1] has defined Hankel transform of functions of matrix argument by

$$
g(\Lambda)=\int A_{c}(\wedge R)(\operatorname{det} R)^{c} f(R) d R \text {, }
$$

where $A_{c}$ is the Bessel function of matrix argument, and $f \in L_{c}^{2}, L_{c}^{2}$ being the Hilbert space of functions for which the norm defined by

$$
\|f\|_{C}^{2}=\int_{R>0}|f(R)|^{2}(\operatorname{det} R)^{c} d R \text {, }
$$

where $c$ is a real number greater than $-1 / 2$.

We can find a connection between Hankel transform and generalized Laplace transform The result can be stated in the form of the following theorem.

THEOREM 7. If $\mathrm{f}(\Lambda) \in \mathrm{L}_{c-p}^{2}$, and

$$
g(z)=\frac{\Gamma_{m}(a)}{\Gamma_{m}(b)} \int_{\Lambda>0} F_{1}(a ; b ;-\wedge Z)(\operatorname{det} \wedge)^{b-p_{f}} f(\wedge) d \wedge,
$$

converges absolutely in the simply connected region $\operatorname{Re}(a), \operatorname{Re}(b)>p-1$ for $Z>0$, and

$$
\phi(Z)=\int_{\Lambda>0}^{A_{b-p}}(\Lambda Z)(\operatorname{det} \wedge)^{b-p_{f}} f(\Lambda) d \wedge,
$$

then

$$
\operatorname{Lim}_{a \rightarrow \infty} \frac{1}{\Gamma_{m}(a)} g\left(\frac{1}{a} Z\right)=\phi(Z) .
$$

The proof of the theorem is quite simple in view of the limit (see Herz [1]):

$$
\operatorname{Lim}_{a \rightarrow \infty} F_{1}\left(a ; b ;-\frac{1}{a} R\right)=\Gamma_{m}(b) A_{b-p}(R) \text {. }
$$

We shall now prove a theorem which serves as real inversion theorem for the generalized Laplace transform.

THEOREM 8. If

$$
g(a ; b ; z)=\frac{\Gamma_{m}(a)}{\Gamma_{m}(b)} \int_{\wedge>0} F_{1}(a ; b ;-N)(\operatorname{det} \wedge)^{b-p} f(\wedge) d \wedge
$$

is absolutely convergent for $a>p-1, b>p-1, z>0$, and $f, g \in L_{b-p}^{2}$; then

$$
f(N)=\lim _{a \rightarrow \infty} \frac{a^{b m}}{\Gamma_{m}(a)} \quad H\{g(a ; b ; \wedge a)\}
$$

where

$$
H\{g(a ; b ; a Z)\}=\int_{Z>0} A_{b-p}(a \wedge Z)(\operatorname{det} Z)^{b-p} g(a ; b ; Z) d Z .
$$

PROOF. Since $f \in \mathrm{L}_{\mathrm{b}-\mathrm{p}}^{2}$ it's Hankel transform will exist. So we have

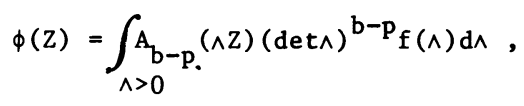

Now, from Theorem 7, we have 


$$
\phi(Z)=\lim _{a \rightarrow \infty} \frac{1}{\Gamma_{m}(a)} \cdot g\left(a ; b ; \frac{1}{a} Z\right)
$$

By the Hankel inversion of (5.8) and (5.9), we obtain

$$
f(\wedge)=\lim _{a \rightarrow \infty} \frac{1}{\Gamma_{m}(a)} \quad A_{b-p}(\wedge Z)(\operatorname{det} Z)^{b-p} g\left(a ; b ; \frac{1}{a} Z\right) \text {. }
$$

Now, changing variables from $Z$ to $a z$, and noting that the Jacobian of transformation for the change is (a) ${ }^{\mathrm{pm}}$, we have the desired result.

\section{REFERENCES}

1. HERZ, C. S., Besse1 functions of matrix argument, Annals of Mathematics, $\underline{61}$ (1955), 474-523.

2. JOSHI, J. M. C., Inversion and representation theorems for a generalized Laplace transform, Pacific Journal of Mathematics, 14(1965) 977-985.

3. GARDING, L., The solution of Cauchy's problem for two totally hyperbolic linear differential equations by means of Riesz integrals, Annals of Mathematics, 48 (1947) 785-826.

4. ROONY, P.G., A generalization of complex inversion formula for the Laplace transformation, Proc. Amer. Math. Soc., 5(1954) 385-391.

5. YOSHIDA, K., Functional Analysis, Narosa Publishing HOuse, New Delhi (1979). 


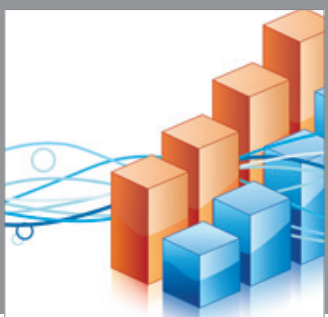

Advances in

Operations Research

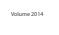

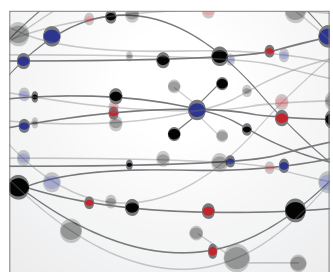

\section{The Scientific} World Journal
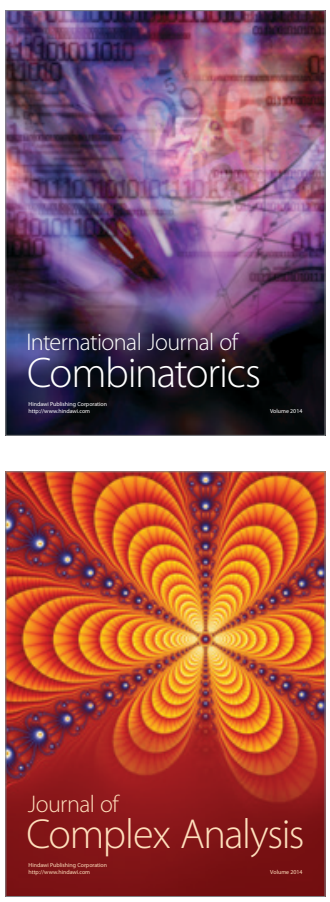

International Journal of

Mathematics and

Mathematical

Sciences
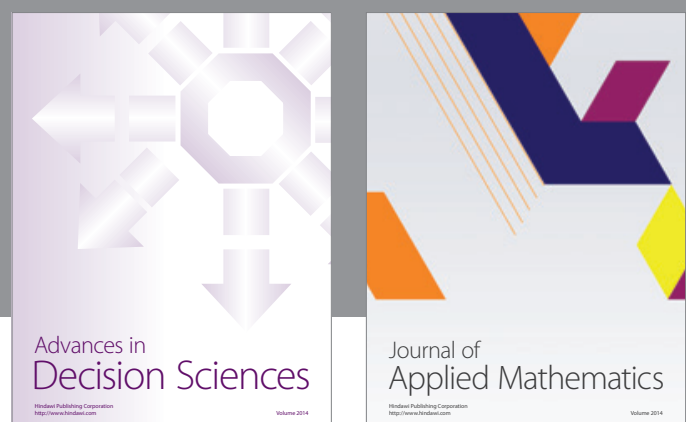

Journal of

Applied Mathematics
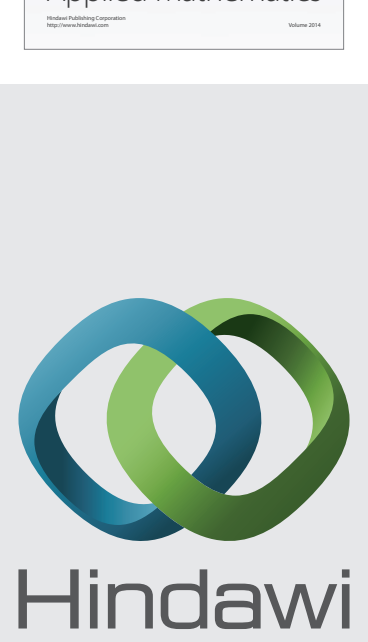

Submit your manuscripts at http://www.hindawi.com
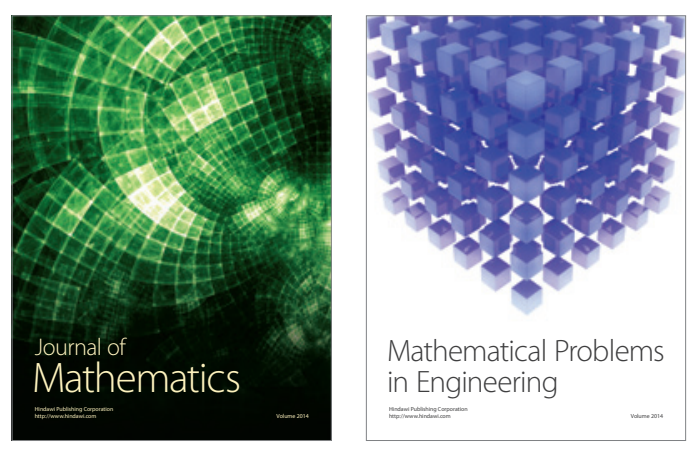

Mathematical Problems in Engineering
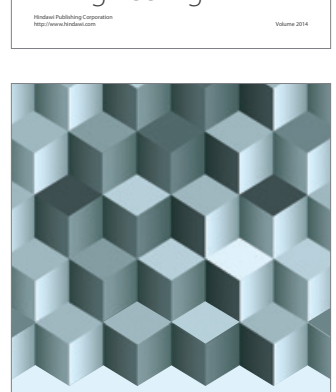

Journal of

Function Spaces
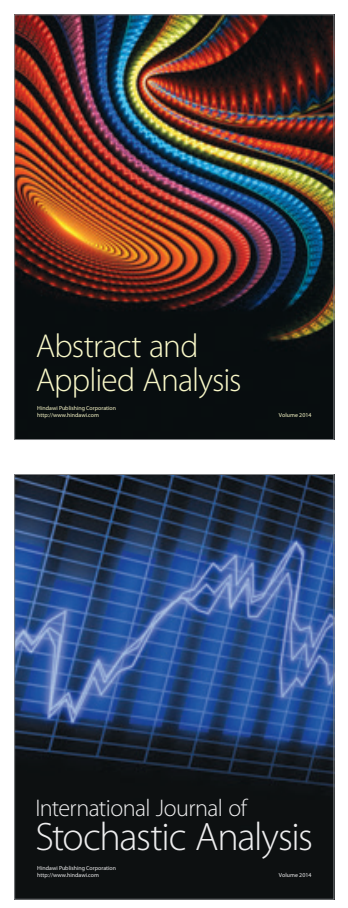

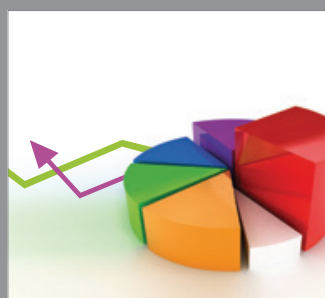

ournal of

Probability and Statistics

Promensencen
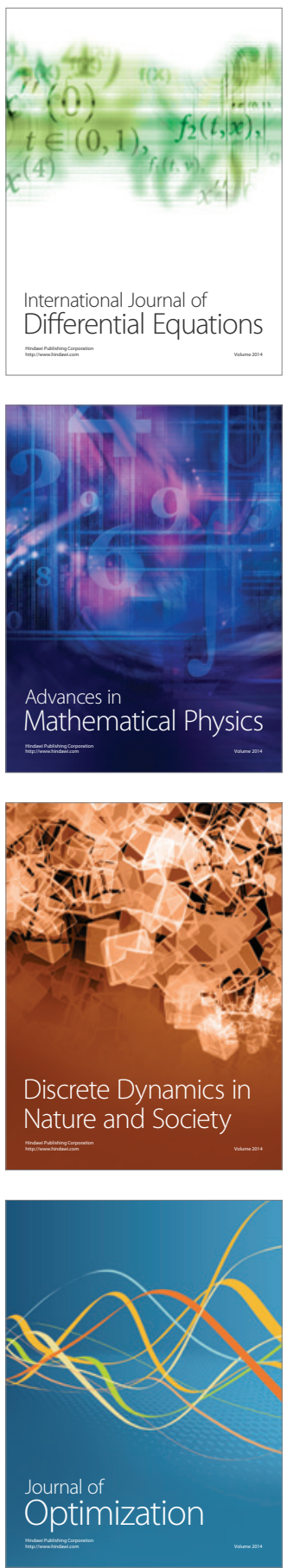